\title{
Acute EBV infection and HIV antibody cross- reactivity in a first Time donor
}

\begin{abstract}
An 18-years-old female college student was screened as a first-time blood (plateletpheresis) donor and was found to have a highly positive HIV Ab ChLIA (duplicate) test. Negative Western Blot (WB), HIV p24 antigen and NAT HCV RNA/ HIV RNA/HBV DNA assays confirmed that the donor was not infected with HIV. The use of other laboratory tests revealed an acute Epstein Barr infection with positive EBV Viral Capsid Antigen (VCA IgM and VCA IgG) antibodies and also positive Heterophile antibodies. Nonspecific reactivity (false-positive) results of HIV infection, by serological tests, may represent a cross-reaction with other human viruses.
\end{abstract}

Keywords: nonspecific reactivity of HIV, EBV infection, blood donor

\author{
Volume I Issue 4 - 2015
}

Kavallierou L,' Bazigou F,' Cheropoulou $A^{2}$

'Blood Transfusion Service Amalia Fleming Hospital, Greece

${ }^{2}$ Blood Transfusion Service Sismanoglio Hospital, Greece

Correspondence: Lilian Kavallierou, Blood Transfusion Service Amalia Fleming Hospital, 20 Koudouriotou Street, 15 | 26 Melissia, Athens, Greece, Tel 210-6139870,

Email liliankavallierou@hotmail.com

Received: November 08, 2015 | Published: December 07, 2015
Abbreviations: ChLIA, chemiluminescent immunoassay; NAT, nucleic acid test; EBNA, epstein-barr nuclear antigen; EA, early antigen

\section{Introduction}

To donate blood, individuals must be at least 18 years old, healthy, and feeling well on the day of donation. Donors are also screened for disease risk factors using a health history questionnaire. Laboratory testing of donated blood prior to transfusion is intended to ensure that recipients receive the safest possible blood products. Blood donation screening for HIV 1 and 2, HBsAg, HCV, HTLV-I/II and Syphilis reveals the presence of infectious markers. ${ }^{1} \mathrm{~A}$ chemiluminescent immunoassay (ChLIA) is used for the qualitative detection of antibodies to HIV 1 and 2, HBsAg, HCV, HTLV-I/II. Moreover, a triplex nucleic acid test (NAT) includes the detection of HBV DNA, HIV-1 RNA and HCV RNA. NAT has reduced the HBV windowperiod from infection to detection by about 8 to 10 days, has closed the window period between infection and detection of antibodies for those infected with HIV, by about 2 weeks, and for those infected with HCV by about 50 to 60 days. ${ }^{2,3}$ Blood based HIV serological assays have a demonstrated sensitivity between $99.3 \%$ to $99.7 \%$, and specificity between $99.91 \%$ and $99.97 \%$. Moreover, the specificity of fourth-generation HIV assays is between $99.50 \%$ and $99.90 \%{ }^{4}$ Today, the false negative rate, for HIV antibody detection, in the general U.S. population is around $0.003 \%$, or three times out of every 100,000 tests. False positive rates are far lower, between $0.0004 \%$ and $0.0007 \%$, due to the practice by which the initial positive result is confirmed by a WB test. ${ }^{5}$ False-positive HIV screening results could cause substantial psychological distress while waiting for a confirmatory test. Blood transfusion services have the responsibility to provide accurate results, information, counseling, and support, to help donors understand and respond to unexpected information about their health status.

\section{Case report}

An 18-years-old sexually active female college student was screened as a first-time donor, willing to donate apheresis platelets. This donor was free of symptoms, reported no significant HIV risk activities, and had not had a recent influenza or hepatitis B vaccination. Her 4th generation ChLIA HIV assay duplicate test $(+$ new sample) was highly positive (39.2s/co). Negative viral load (NAT HIV-1 RNA), WB and HIV p24 antigen (single) tests confirmed that the donor was not infected with HIV. The use of other laboratory tests revealed an acute Epstein Barr (EBV) infection with the EBV Viral Capsid Antigen (VCA IgM and VCA IgG) antibodies positive, Monospot test positive and the Epstein-Barr Nuclear Antigen (EBNA$1 \mathrm{IgG})$ antibodies negative. Furthermore, IgM antibodies for CMV, HSV-1 and HSV-2 were negative. The blood donor's screening and laboratory testing is as summarized in Table 1. The blood donor was given information, provided with counseling, and was barred from further blood donations on a temporary basis.

Table I Blood donor's screening and laboratory testing

\begin{tabular}{ll}
\hline Test & Result \\
\hline HIV-I/2 ELISA/CHLIA duplicate test + new sample & Highly Positive \\
HIV RNA/HCV RNA/HBV DNA NAT & Negative \\
WESTERN BLOT HIV, p24 HIV Ag & Negative \\
HbsAg, HCV Ab, HTLVI/II Ab, RPR & Negative \\
Anti HBc, anti HBe, IgM antiHBc, HBe Ag, anti HBs & Negative \\
EBV viral capsid antigen (VCA) antibody & Positive (IgM) \\
EBV viral capsid antigen (VCA) antibody & Positive ( IgG) \\
EBV nuclear antigen (EBNA) antibody & Negative (lgG) \\
Heterophile antibody (Monospot) & Positive \\
CMV Ab & Negative (lgM) \\
HSV IAb, HHVIAb & Negative (IgM) \\
HSV 2Ab, HHV2Ab & Negative (IgM) \\
\hline
\end{tabular}

\section{Discussion}

Evidently, the case study blood donor has an acute mononucleosis (IM) infection and not HIV. IM is usually a self- limiting infection, most often caused by EBV, which is a human herpes virus. IM is particularly common in populations with many young adults, such as active-duty military personnel and college students. EBV IM 
can be diagnosed using a variety of unrelated non-EBV heterophile antibodies (e.g. Monospot) and specific EBV antibodies. There is a high agreement between the heterophile antibody tests and the VCAIgM ELISA, but the latter are more sensitive. Although virtually $100 \%$ specificity exists with the Monotest, other disorders may rarely produce a nonspecific reactivity (false-positive) result, such as infection, malignancy and connective tissue diseases.

The blood donor's positive Monospot test and EBV-specific antibody pattern (positive VCA IgM and VCA IgG anti-EBV antibodies) is consistent with an acute EBV infection. IgM and IgG antibodies directed against the VCA of EBV are useful in confirming the diagnosis of EBV and in differentiating acute and/or recent infection from previous infection. For clinical purposes, the most useful EBV-specific antibodies are the VCAs and the EBNAs. Both VCA and EBNA antibodies are usually reported as IgM or IgG antibodies. Acute infection is diagnosed in patients who have an increased EBV IgM VCA titer. Following acute infection, the increase in IgM VCA antibody titers peaks after 4-8weeks, decrease in most patients after 3-6months but may persist in low titer for up to one year. Later in the course of infection, the increase in IgM VCA antibodies may be accompanied by an increase in IgG VCA antibodies and an increase in IgG EBNA antibodies. EBV IgG VCA antibodies, rise later than the IgM VCA antibodies, as mentioned, but remain elevated with variable titers for life. Many laboratories report EBNA titers only, which usually measure the IgG EBNA, these antibodies appear after 1-2months and persists throughout life. The presence of these antibodies suggests previous exposure to the antigen (past infection), which may have been subclinical or clinical, and excludes EBV infection acquired in the previous year, as in the case of our blood donor, where her EBNA (IgG) antibodies were negative. Other antigens indicating EBV infection are less useful diagnostically and include early antigen (EA), which is present early in EBV infectious mononucleosis. ${ }^{6-8}$ Positivity of the HIV test was presumed to reflect cross-reactivity (false positive), in the setting of acute EBV infection, as confirmed by negative WB, p24 antigen and NAT HIV-1 RNA assays.

Within a general population, the rate of false negatives and false positives is largely determined by the sensitivity and specificity of the test used. Although the rate of false positive and false negative HIV test results are low, they do sometimes occur. Their incidence is largely influenced by a number of factors, including the type of test used, the limitations of current testing technologies and even the time at which a person is given a test. Specifically, a false positive occurs when an HIV test incorrectly identifies a non-HIV-antibody as being an HIV antibody because antigens similar to HIV are detected. Various foreign antigens and infectious agents produce antibodies that nonspecifically cross-react with antigens in some HIV test kits. The rate of false positive results obtained with blood donors varies from 0.2 to $0.8 \%$, depending on the donor's background.

A common cause of false positive serologic screens for HIV is recent influenza vaccination or an incidental viral infection. In addition to flu vaccination and viral infections, false-positive HIV immunoassay results have been reported in a variety of other conditions, such as autoimmune disease, renal failure, cystic fibrosis, multiple pregnancies, blood transfusions, liver diseases, parenteral substance abuse, hemodialysis, and vaccinations against hepatitis B and rabies. ${ }^{9-13}$ This should be noted by medical practitioners and labs that conduct screening tests in order to counteract traumatic social impasse. A false diagnosis of HIV infection can be proved by the combination of EIA, WB and NAT testing in blood donor. Individuals with a positive or indeterminate WB result should be further evaluated by RNA PCR or NAT testing and HIV serologic analysis on a followup sample. Serologic testing for transfusion transmitted diseases had historically been the foundation of blood screening, while newer strategies, as NAT, have helped further shorten the "window period". In addition to ELISA, NAT is being increasingly used in many countries to further improve blood safety. On the other hand, technical errors do occur and although they cannot be eliminated totally, they can be minimized through the institution of a thorough quality assurance program and documented preventive measures. Clerical errors are the most common and can be addressed effectively through a dedicated supervisory review mechanism. Several essential quality assurance measures are outlined subsequently. Therefore, to ensure the quality of screening blood tests, a program consisting of quality assurance, quality control and quality assessment is necessary.

\section{Conclusion}

Screening of donors is recommended for the provision of a safe blood supply. Methods performed to identify the presence of HIV include a highly sensitive and specific anti HIV-1/2 immunoassay or HIV combination antigen-antibody immunoassay (EIA/ChLIA). All sera repeatedly positive by the above methods should be submitted to WB confirmatory testing analysis. NAT also adds the benefit of resolving false reactive donations on serological methods which is very important for donor notification and counseling. Nonspecific reactivity (false-positive) results of HIV infection, by serological tests, may represent a cross-reaction with other human viruses. Physicians and Transfusion Service Personnel should be aware of specific causes of inaccurate results.

\section{Acknowledgements}

None.

\section{Conflict of interest}

The author declares no conflict of interest.

\section{References}

1. WHO. Screening Donated Blood for Transfusion-Transmissible Infections. WHO; 2010.

2. Weusten J, Vermeulen M, Van Drimmelen H, et al. Refinement of a viral transmission risk model for blood donations in seroconversion window phase screened by nucleic acid testing in different pool sizes and repeat test algorithms. Transfusion. 2010;51(1):203-215.

3. Hans R, Marwaha N. Nucleic acid testing-benefits and constraints. Asian J Transfus Sci. 2014;8(1):2-3.

4. Constantine N. HIV Antibody Assays. University of Maryland School of Medicine, Maryland, USA: HIV In Site Knowledge Base Chapter; 2016.

5. Kleinman S, Busch MP, Hall L, et al. False-positive HIV-1 test results in a low-risk screening setting of voluntary blood donation. JAMA 1998;280(12):1080-1085.

6. Cunha BA, Bronze MS. Infectious mononucleosis workup; 2014.

7. Ebell MH. Epstein-barr virus infectious mononucleosis. Am Fam Physician. 2004;70(7):1279-1287.

8. De Paschale M, Clerici P. Serological diagnosis of Epstein-Barr virus infection: Problems and solutions. World J Virol. 2012;1(1):31-43. 
9. Mackenzie W, Davis J, Peterson D, et al. Multiple false-positive serologic tests for HIV, HTLV-1 and hepatitis C following influenza vaccination, JAMA. 1992;268(8):1015-1017.

10. Scarano A. Causes of a false positive HIV test; 2011.

11. Lee D, Eby W, Molinaro G. HIV false positivity after hepatitis B vaccination. Lancet. 1992;339(8800):1060.
12. Ladizinski B, Sankey C. Acute Epstein-Barr virus infection and human immunodeficiency virus antibody cross-reactivity. Am J Med. 2014;127(6):e9-e10.

13. Amechi BO, Osagie RN, Chikwendu CI. Evaluation of false positivity and cross reactivity in the investigation of human immunodeficiency virus (HIV) antibodies. International Journal of Community. 2012;1(2):54-59. 